been made in the period prior to the sampling, and there is no drywall in these wards. However, the presence of this material in other wards or hospital common areas is not excluded. The presence of $N$. hiratsukae on the computer keyboard and printer in the medical stations suggests that it may have been spread by healthcare professionals.

Unfortunately, the rapid evolution of the COVID-19 epidemic in Italy shortly after our environmental sampling prevented further control samplings until 6 months later, when no N. hiratsukae were isolated. A more accurate sanitation during COVID-19 epidemic has probably reduced the presence of possible pathogens on the surfaces.

Despite the presence of $N$. hiratsukae in the environment of the sampled ICUs, no clinical isolates of $N$. hiratsukae were detected in the hospital in the same period, even if it cannot be ruled out due to the difficulties in identifying this species. Indeed, macroscopic and microscopic identification is not specific, and methods such as MALDI, in use in the hospital microbiology laboratories, do not identify this species. Neosartorya infections are probably underreported due to the aforementioned difficulties in identification and to the laboratory practice of discarding the nonsporulating or slowly sporulating white mycelia as laboratory contaminants.

Variable MIC values for N. hiratsukae are reported in the literature, showing mainly azole-susceptible patterns ${ }^{2,5,6}$ and some sporadic high itraconazole MIC values. ${ }^{6}$

Preventing nosocomial infections is very important, especially for ICU patients. Environmental control through environmental sampling of air and surfaces represents a valid tool that should be performed systematically, not only in the case of an outbreak. Laboratory routine methods for the identification are not sufficient to perform the correct identification of $N$. hiratsukae, obtainable only with molecular biology techniques. The correct identification of $N$. hiratsukae and other Neosartorya spp as well as their antifungal susceptibilities should be further investigated.
Acknowledgments. The authors would like to thank Dr Romualdo Grande for his communication and the hospital staff of the ICU involved in this study for their collaboration.

Financial support. No financial support was provided relevant to this article.

Conflicts of interest. All authors report no conflicts of interest relevant to this article.

\section{References}

1. Udagawa S, Tsubouchi H, Horie Y. Neosartorya hiratsukae, a new species of food-borne Ascomycetes. Trans Mycol Soc Jpn 1991;32:23-29.

2. Guarro J, Kallas EG, Godoy P, et al. Cerebral aspergillosis caused by Neosartorya hiratsukae, Brazil. Emerg Infect Dis 2002;8:989-991.

3. Predari SC, de Paulis AN, Veron D, Zucchini A, Santoianni JE. Fungal peritonitis in patients on peritoneal dialysis: twenty-five years of experience in a teaching hospital in Argentina. Rev Argent Microbiol 2007;39:213-217.

4. Shivaprakash MR, Jain N, Gupta S, Baghela A, Gupta A, Chakrabarti A. Allergic fungal rhinosinusitis caused by Neosartorya hiratsukae from India. Med Mycol 2009;47:317-320.

5. Mellado E, Alcazar-Fuoli L, Garcia-Effron G, Alastruey-Izquierdo A, Cuenca-Estrelal M, Rodriguez-Tudela JL. New resistance mechanisms to azole drugs in Aspergillus fumigatus and emergence of antifungal drugsresistant A. fumigatus atypical strains. Med Mycol 2006;44:S367-S371.

6. Koutroutsos K, Arabatzis M, Bougatsos G, Xanthaki A, Toutouza M, Velegraki A. Neosartorya hiratsukae peritonitis through continuous ambulatory peritoneal dialysis. J Med Microbiol 2010;59:862-865.

7. Rodriguez-Tudela JL, Arendrup MC, Arikan S, et al. European Committee on Antimicrobial Susceptibility Testing. EUCAST definitive document E.DEF 9.1: Method for Determination of Broth Dilution Minimum Inhibitory Concentrations of Antifungal Agents for Conidia-Forming Moulds. Växjö, Sweden: EUCAST; 2008.

8. Antifungal agents' breakpoint tables for interpretation of MICs-version 10.0. European Committee on Antimicrobial Susceptibility Testing website. https://www.eucast.org/fileadmin/src/media/PDFs/EUCAST_files/AFST/ Clinical_breakpoints/AFST_BP_v10.0_200204.pdf. Accessed March 25, 2021.

9. Andersen B, Dosen I, Lewinska AM, Nielsen KF. Pre-contamination of new gypsum wallboard with potentially harmful fungal species. Indoor Air 2017; 27:6-12.

\title{
Coronavirus disease 2019 (COVID-19) vaccination in healthcare workers: An early real-world experience
}

\author{
Andrew P. Jameson MD, FACP ${ }^{1,2}$ (1), Tara Sebastian BS $^{1}$ and Liberty R. Jacques DNP, RN, CIC \\ ${ }^{1}$ Michigan State University College of Human Medicine, Grand Rapids, Michigan and ${ }^{2}$ Mercy Health Saint Mary's Hospital, Grand Rapids, Michigan
}

To the Editor-The spread of the novel severe acute respiratory syndrome coronavirus 2 (SARS-CoV-2) and its associated coronavirus disease (COVID-19) has affected millions of people worldwide. As of February 2021, the pandemic had claimed the lives of $>2.4$ million individuals. ${ }^{1}$ Highly effective vaccines against the SARS-CoV-2 virus have attracted intense attention as an attainable solution to mitigate further spread.

\footnotetext{
Author for correspondence: Andrew P. Jameson, E-mail: andrew.jameson@mercyhealth. com

Cite this article: Jameson AP, Sebastian T, and Jacques LR. (2022). Coronavirus disease 2019 (COVID-19) vaccination in healthcare workers: An early real-world experience. Infection Control \& Hospital Epidemiology, 43: 950-951, https://doi.org/10.1017/ ice. 2021.171
}

One such vaccine is the BNT162b2 mRNA vaccine, first described by Polack et al, ${ }^{2}$ now being produced and distributed by Pfizer (New York City, NY). In December 2020, data from phase 2 and 3 clinical trials with 36,523 participants were published describing the initial efficacy of the vaccine in preventing symptomatic COVID-19 transmission. These data suggested that, after 1 dose, the BNT162b2 vaccine had an efficacy of $52 \%$. At 7 days after the second dose, the vaccine showed a highly promising efficacy of $95 \%{ }^{3}$

Here, we present data from a vaccination program for healthcare workers (HCWs) of a medium-sized urban hospital in the Midwest region in the United States. Throughout the vaccination effort, the community burden of COVID-19 remained concerningly high, with 
13,604 total new cases among the county's $\sim 657,000$ residents between December 15, 2020, and March 24, 2021.4,5

Beginning on December 17, 2020, a voluntary vaccination program consisting of 2 doses of the BNT162b2 vaccine was offered to all hospital staff. The vaccine was administered using standard protocol according to the emergency use authorization issued by the FDA.

During this period, HCWs continued to participate in daily screening for symptoms of COVID-19. Any employee experiencing concerning symptoms was referred to complete nasopharyngeal swab testing for COVID-19 before returning to work. Testing was offered daily to create easy access and was completed via nasopharyngeal swab utilizing the Abbott ID Now platform (Abbott Diagnostics, Abbott Park, IL). If an employee tested positive, details of their vaccination status, symptoms, and potential exposures were documented.

Between December 17, 2020, and March 24, 2021, a total of $4,318 \mathrm{HCWs}$ were eligible to receive vaccination. Approximately 3,023 were vaccinated from this group, and 1,295 individuals opted not to pursue vaccination. Moreover, $90 \mathrm{HCWs}$ tested positive for SARS-CoV-2 after a positive daily system screener. Of those individuals, 71 had not received SARS-CoV-2 vaccination, but 19 of these individuals had been vaccinated and 6 individuals from the vaccinated group had received both vaccinations.

Unvaccinated HCWs were significantly more likely to test positive for SARS-CoV-2 than those vaccinated, with an odds ratio (OR) of 8.7 (95\% confidence interval [CI], 5.2-14.5; $P<.0001$ ). The number needed to vaccinate to prevent 1 symptomatic COVID-19 diagnosis in this period was 20.6. Full immunity is not reached until 7 days after the second dose of BNT162b2, and 5 individuals tested positive for SARS-CoV-2 after reaching this full immunity. For those who tested positive after receiving only 1 dose of the vaccine, the average time from vaccination to test positivity was 9.9 days (range, 5-15). For those who received both doses, the average time from the second vaccine administration to test positivity was 25.5 days (range, 7-58 days). Unvaccinated colleagues had 33.1 times greater odds of testing positive for SARS-CoV-2 than those who had reached full immunity (OR, 33.1; 95\% CI, 13.6-82.3; $P<.0001$ ).

It is not surprising that fewer infections occurred among vaccinated HCWs compared to those HCWs who declined vaccination in a community with a high rate of new COVID-19 infections. Many organizations have implemented a voluntary vaccination program, and results like these emphasize the need to incentivize employees from any industry to get the SARS-CoV-2 vaccine. Odds ratios of 8.7 for any vaccine status and 33.1 for full vaccine protection demonstrate the significant risk reduction provided by the vaccine.
Despite the protection afforded by the vaccine, it is concerning that many individuals tested positive after receiving 1 or 2 doses of the vaccine. This finding seems to stress the importance of not only encouraging vaccination itself but also encouraging the continued adherence to well-established mitigation strategies.

The research detailed in this letter has several limitations. The voluntary nature of the vaccine program had the potential to select for individuals whose behaviors might place them at decreased risk of COVID-19 acquisition regardless of vaccination. Additionally, the fluctuating nature of employees over a 3-month period made it very difficult to get an accurate count of total employees and total vaccinations. Finally, the imperfect nature of PCR testing does raise the possibility that an individual may not be actively infected with SARS-CoV-2 yet may demonstrate ongoing shedding from a remote infection. Although this is less likely because we only tested symptomatic individuals, without cycle thresholds or cell culture it is difficult to be certain about active infection.

In conclusion, COVID-19 infection has resulted in a considerable burden and cost to the healthcare organizations delivering care in the United States. Vaccination provides a proactive approach to decreasing this burden, and a number needed to vaccinate to prevent 1 infection of 20.6 is a very strong motivating factor for organizations to maximize their employee vaccine uptake. Robust education, debunking myths, and easy access to vaccination will be essential for employers from any industry to encourage vaccination among their workforce. For organizations that provide paid time off for those infected with COVID-19, the improved safety and well-being of the workforce has the added benefit of potential cost savings.

Acknowledgments. We acknowledge Jill Jameson for editing manuscript.

Financial support. No financial support was provided relevant to this article.

Conflicts of interest. All authors report no conflicts of interest relevant to this article.

\section{References}

1. Mortality analyses. Johns Hopkins Coronavirus Resource Center website. https//coronavirus.jhu.edu/data/mortality. Accessed March 1, 2021.

2. Polack FP, Thomas SJ, Kitchin N, et al. Safety and efficacy of the BNT162b2 mRNA COVID-19 vaccine. N Engl J Med 2020;383:2603-2615.

3. Walsh EE, Frenck RW, Falsey AR, et al. Safety and immunogenicity of two RNA-based COVID-19 vaccine candidates. N Engl J Med 2020;383: 2439-2450.

4. COVID-19 data, Kent County, Michigan. accessKent website. https://www. accesskent.com/Health/covid-19-data.htm. Accessed March 1, 2021.

5. QuickFacts: Kent County, Michigan. US Census Bureau website. https:// www.census.gov/quickfacts/kentcountymichigan. Accessed March 1, 2021. 